\title{
ISN and the 'new nephrology'
}

\author{
William G Couser
}

I recently met with over 30 leaders of the 72 national societies with which the International Society of Nephrology (ISN) is affiliated. I was particularly struck by the implication of one question: "What is the ISN doing to promote the 'new nephrology'?"

The 'new nephrology' was born of the seminal observation that progression of kidney disease is propelled by modifiable hemodynamic factors (Hostetter TH et al. [1982] Am J Med 72: 375). Subsequent K/DOQI classification of stages of chronic kidney disease (CKD) indicated that about $10 \%$ of the general population in several countries have CKD (National Kidney Foundation [2002] Am $J$ Kidney Dis 39: S1). Finally, patients with CKD were shown to be at increased risk of developing cardiovascular disease (Go AS et al. [2004] New Engl J Med 351: 1296); early treatment could slow progression and could significantly reduce the incidence of cardiovascular events (Asselbergs FW et al. [2004] Circulation 110: 2809).

What, then, is the ISN doing to address this issue? All core ISN programs, now encompassed by COMGAN, have been expanded and restructured to emphasize early detection and prevention. The Fellowship Program has expanded by $25 \%$, and shifted its focus from basic science research to epidemiology and clinical research. Affiliations with centers that can formally teach such skills have been strengthened. Similarly, the Sister Renal Centers program now has a stronger emphasis on links between centers that can promote clinical research training and performance of studies in the developing world. The John Dirks Fellowship in Clinical Research has been added to the portfolio. Continuing medical education programs and site visits in developing countries now more often incorporate a lecture on detection and prevention of kidney disease, plus a visit by the ISN and local speakers to government health officials.

The ISN Research and Prevention Committee now sponsors ongoing programs in ten countries, which aim to prove that prevention can be effective
All core ISN

programs, now

encompassed

by COMGAN,

have been

expanded and

restructured

to emphasize

early detection

and prevention.

WG Couser is

President of the

International Society

of Nephrology.

\section{Competing interests}

The author declared he has no competing interests.

www.nature.com/clinicalpractice doi:10.1038/ncpneph0302 and cost-efficient in the developing world. The committee developed a global early detection and intervention template for emerging countries that encompasses screening and management phases, and data assessment. Applications for research grants are now accepted from groups in disadvantaged areas that seek to implement such programs. The program template can be downloaded from the ISN website.

Since 2003, the ISN has sponsored 16 2-day prevention meetings. Local providers are helped to present data on kidney disease from their areas, and position papers are developed that can be used to approach governments.

The ISN Medical Advisory Committee reaches out to international societies of hypertension, diabetes, cardiovascular disease and transplantation with the aim of coordinating joint approaches to prevention of kidney and cardiovascular disease in high-risk populations and regions. There is a plan to interact in a more formal way with non-physician members of renal care teams who will be central to implementation of large-scale prevention efforts.

The most important ongoing initiative is World Kidney Day. Together with the International Federation of Kidney Foundations, we tentatively launched World Kidney Day on 9 March 2006; the response was overwhelming. World Kidney Day 2007 will be celebrated on 8 March and will be promoted even more widely than the fledgling 2006 effort. By attracting media and public attention, such events stimulate individuals and primary care physicians to think about CKD screening, and broadcast the compelling message to government health-care officials that both lives and money can be saved.

I do not want to leave you with the impression that the ISN is abandoning its traditional focus on basic science and research; the new 'bench to bedside' Nexus meetings (e.g. one on bone disease in Copenhagen this month) prove that this is not the case. Nonetheless, much of the science has been done and the opportunities for translation are enormous. 